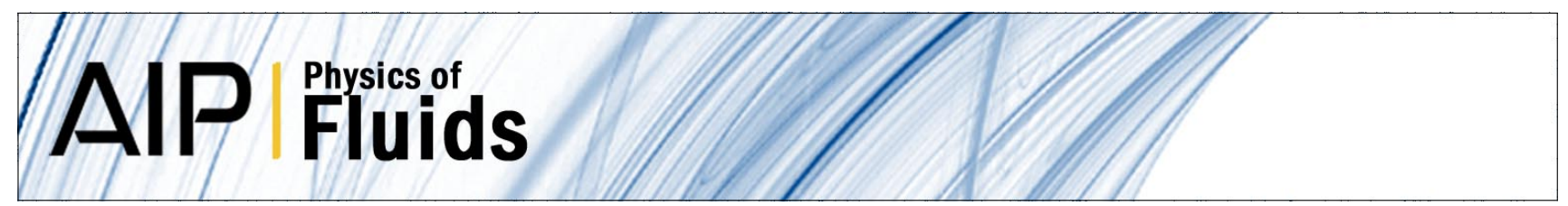

\title{
Effect of a splitter plate on the dynamics of a vortex pair
}

Jason Rabinovitch, Vincent Brion, and Guillaume Blanquart

Citation: Phys. Fluids 24, 074110 (2012); doi: 10.1063/1.4737878

View online: http://dx.doi.org/10.1063/1.4737878

View Table of Contents: http://pof.aip.org/resource/1/PHFLE6/v24/i7

Published by the American Institute of Physics.

\section{Related Articles}

Detuned resonances of Tollmien-Schlichting waves in an airfoil boundary layer: Experiment, theory, and direct numerical simulation

Phys. Fluids 24, 094103 (2012)

Effect of plate thickness on particle deposition velocity onto a face-up flat plate situated parallel to an airflow J. Appl. Phys. 110, 063518 (2011)

Traveling wave-induced aerodynamic propulsive forces using piezoelectrically deformed substrates Appl. Phys. Lett. 99, 114102 (2011)

Effects of continuum breakdown on hypersonic aerothermodynamics for reacting flow Phys. Fluids 23, 027101 (2011)

Evaluation of active flow control applied to wind turbine blade section

J. Renewable Sustainable Energy 2, 063101 (2010)

\section{Additional information on Phys. Fluids}

Journal Homepage: http://pof.aip.org/

Journal Information: http://pof.aip.org/about/about_the_journal

Top downloads: http://pof.aip.org/features/most_downloaded

Information for Authors: http://pof.aip.org/authors

\section{ADVERTISEMENT}

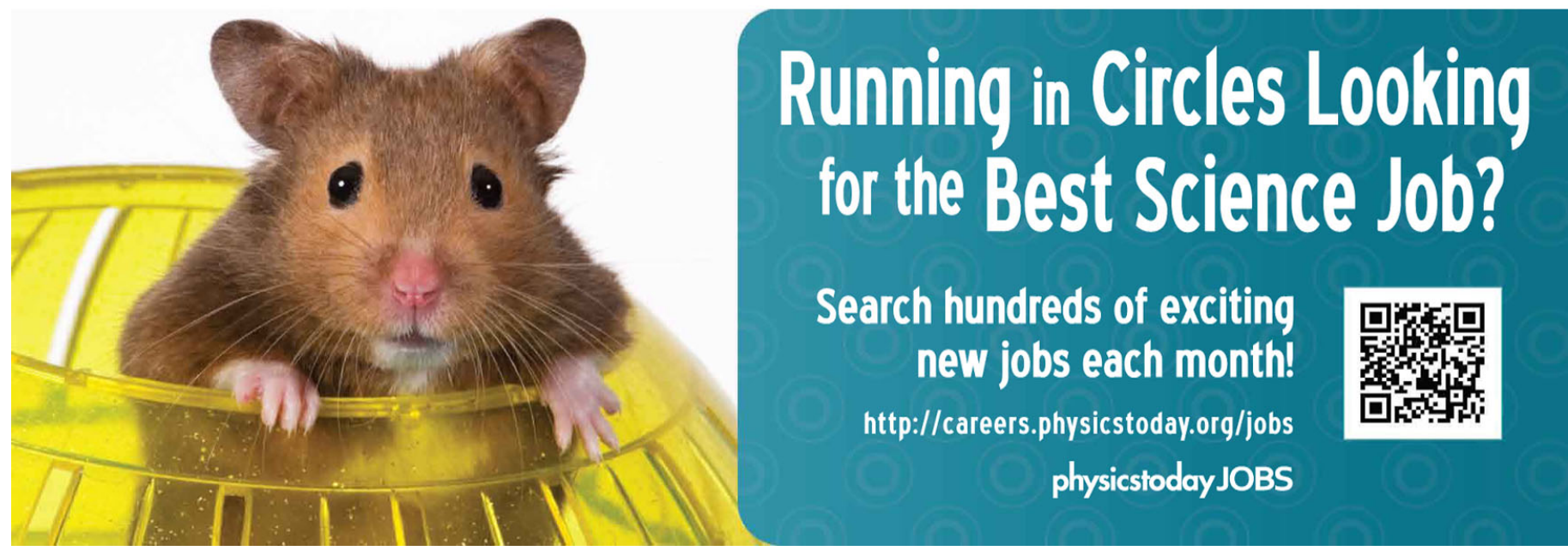




\title{
Effect of a splitter plate on the dynamics of a vortex pair
}

\author{
Jason Rabinovitch, ${ }^{1, a)}$ Vincent Brion, ${ }^{2}$ and Guillaume Blanquart ${ }^{3}$ \\ ${ }^{1}$ Graduate Aerospace Laboratories, California Institute of Technology, Pasadena, \\ California 91125, USA \\ ${ }^{2}$ ONERA - Departement d'Aerodynamique Fondamentale et Experimentale, \\ 8 rue des Vertugadins, 92190 Meudon, France \\ ${ }^{3}$ Mechanical Engineering Department, California Institute of Technology, \\ Pasadena, California 91125, USA
}

(Received 7 September 2011; accepted 28 June 2012; published online 23 July 2012)

An experimental and numerical study was performed to investigate and compare the behavior of a counter-rotating vortex pair and a single vortex in the vicinity of a wall. This analysis is motivated by the theoretical equivalence, in the inviscid limit, between these two configurations. A wind tunnel with two NACA0012 profiles mounted vertically with an optional splitter plate in the center and a stereoscopic particle image velocimetry system was used to experimentally study these interactions. Many significant differences were found between the two configurations, including the growth of the Crow instability in the two vortex configuration, but not in the one vortex/wall configuration. The numerical results re-enforced the experimental results, and emphasized the fundamental physical differences between the two configurations. While modeling a vortex wall system with an image vortex may give correct integral results for loads experienced by blades, this model does not accurately describe the downstream dynamics of the vortex system. (c) 2012 American Institute of Physics. [http://dx.doi.org/10.1063/1.4737878]

\section{INTRODUCTION}

Counter-rotating vortex pairs have received a lot of attention in the aerodynamic community. The most common occurrence of these vortices are wing-tip vortices, which dominate the behavior of the wake of an airplane. ${ }^{1,2}$ To avoid endangering following aircrafts, a minimum separation time is enforced between takeoffs and landings based on the relative size of the two aircrafts. If the time needed for the wing-tip vortices to decay could be shortened, airport capacity would be greatly increased, while maintaining the same margin of safety.

Two different instabilities occur as a result of the interaction between two counter-rotating vortices: a short-wavelength instability and a long-wavelength instability. The short-wavelength fluctuations are referred to as the Widnall instability, ${ }^{3}$ and the long-wave-length instability is referred to as the Crow instability. ${ }^{4}$ The Crow instability is considered to be the most important instability contributing to the eventual decay of the vortices, and will be the primary focus of this work.

In turbo machinery, the relative motion of the rotor blades and the stationary wall produces a vortex, often referred to as a tip-leakage vortex. The resulting system is similar to the wing-tip vortices produced behind an airplane. Schematics of this process are shown in Fig. 1. Similar to wing-tip vortices, the pressure difference between the suction side and pressure side of the blade induces a flow that eventually rolls up into a single vortex. The characteristics of the vortex are influenced heavily by the aerodynamics of the blade, by the gap between the blade and the wall, and by the relative motion between the rotor casing and the blade. ${ }^{5}$ Previous studies have shown that the mechanism for the formation of a tip-leakage vortex is primarily inviscid. ${ }^{6-8}$ Under these conditions, an image vortex may be used to replace the wall, resulting in a situation similar to trailing wing-tip

\footnotetext{
a)Electronic mail: Jason.Rabinovitch@caltech.edu.
} 


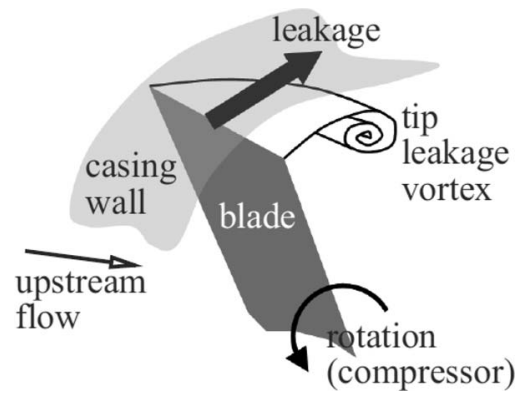

FIG. 1. Schematic photo of a tip-leakage vortex. ${ }^{10}$

vortices. ${ }^{9}$ In a similar manner as for the wing-tip vortices, the Crow instability could be used to encourage vortex breakdown and ultimately increase the efficiency of a compressor.

A variety of experimental techniques have been used to reproduce tip-leakage vortices. For example, a linear cascade has been used, which consists of several blades put side by side, and then attached to a wall. ${ }^{10,11}$ In order to better simulate the relative motion between the blades and the wall, a moving belt is sometimes used in addition. ${ }^{12}$ Other investigations have used full singlestage or multi-stage compressors. ${ }^{13}$ While the flow field is more realistic using a real compressor, measurements become much more complicated compared to using a cascade due to the increased complexity of the geometry. On the numerical side, studies have been done with Reynolds averaged Navier-Stokes simulations, as well as with large eddy simulations. ${ }^{14,15}$

Many similarities between the two-vortex configuration (wing-tip vortices) and the one-vortex configuration (tip-leakage vortex) have been observed in previous studies. ${ }^{8,10,16}$ These similarities were found to be a useful tool for modeling the flow around the blades in a compressor and the loading they experienced. However, these studies focused primarily on the flow close to the blades, and did not consider the downstream evolution of the vortices. To the authors' best knowledge, no detailed work has been done comparing the downstream characteristics of these two configurations.

The purpose of this study is to investigate the Crow instability in two flow configurations, namely with a split wing apparatus (two-vortex configuration) and a single wing with a splitter plate (one-vortex configuration). More specifically, the objective of this study is to compare and contrast the dynamics of a counter-rotating vortex pair and a tip-leakage vortex. This analysis will be performed both experimentally and numerically. In Secs. II-V, the experimental apparatus will be described, followed by the post-processing techniques used to analyze the data taken. An overview of the results obtained (both experimental and numerical) will be presented, along with a discussion of these results.

\section{EXPERIMENTAL METHODOLOGY}

The two-vortex and one-vortex configurations were investigated using particle image velocimetry (PIV) in ONERA's S2L wind tunnel. This section briefly explains the experimental equipment used as well as the vortex center detection methods.

\section{A. Wind tunnel and experimental apparatus}

Figure 2 shows a schematic diagram of the wind tunnel used for all experiments. It is an open circuit, wooden, low-speed wind tunnel, with a circular test section of diameter $1 \mathrm{~m}$. Figure 3

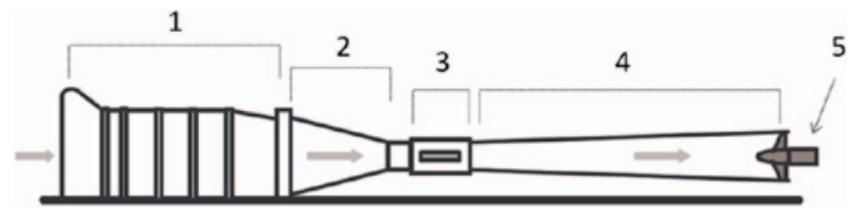

FIG. 2. Schematic drawing of the wind tunnel used. 1-Settling chamber; 2-Converging section; 3-Test section; 4Diffuser; 5-Axial fan. The flow is going from left to right. 


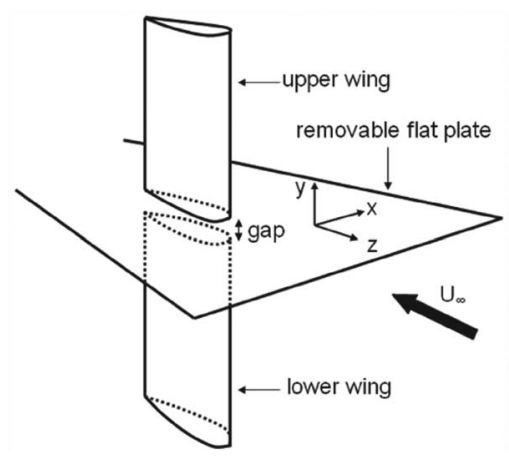

FIG. 3. Basic schematic of the experimental set-up.

shows a basic schematic of the experimental setup found in the test section. The experimental setup consists of two NACA0012 wings mounted vertically in the middle of the test section. This style of setup has been used by several authors before; for example, Devenport et al. ${ }^{17}$ and Heyes et al. ${ }^{18}$ Each wing can be rotated, and the angle of attack can vary between $-15^{\circ}$ and $+15^{\circ}$. In addition, the wings can be moved vertically, so that the gap $(\tau)$ between the wings can be varied from 0 to $50 \mathrm{~mm}$.

Two configurations are possible with this setup as a splitter plate can be added or removed from the test section. Configuration $1(\mathrm{C} 1)$ will refer to experiments where the splitter plate is present. In the $\mathrm{C} 1$ configuration, $\tau$ refers to twice the gap between the airfoil and the splitter plate, in order to remain consistent with the gap size defined for the second configuration. This first configuration is used to study the interaction between a single vortex and a wall. Configuration 2 (C2) will refer to experiments where the splitter plate is not present. This configuration is used to study vortex pair interactions, as two wing-tip vortices are produced and interact with each other. The results from the $\mathrm{C} 1$ configuration are compared and contrasted to the results obtained from the $\mathrm{C} 2$ configuration.

For the present work, the free stream velocity in the test section $\left(U_{\infty}\right)$ is $39.5 \pm 0.5 \mathrm{~m} / \mathrm{s}$. All distances are normalized by the chord length, $c$, and times are normalized by $c / U_{\infty}$. A Reynolds number based on the chord length $\left(R e_{c}\right)$ is defined as

$$
R e_{c}=\frac{U_{\infty} c}{v} .
$$

$v$ is the kinematic viscosity of air, and this corresponds to a Reynolds number of approximately 550000 . A summary of important parameters corresponding to the apparatus is given in Table I.

To ensure that all boundary layers are fully turbulent, carborundum grains were used based on the model given by Schlichting. ${ }^{19}$ Type 80 carborundum grains were placed at $0.25 c$ along the splitter plate, as well as at $0.17 c$ along the airfoils.

Figure 4 shows the general schematic for the PIV system used in this experiment, and a summary of important PIV characteristics is given in the Appendix. For this investigation, measurements were taken at 4 different downstream locations: $2.0 c, 2.5 c, 3.0 c$, and $4.0 c(40 \mathrm{~cm}, 50 \mathrm{~cm}, 60 \mathrm{~cm}$, and $80 \mathrm{~cm}$, respectively). The distance downstream is measured in the direction of the flow, from the

TABLE I. Experimental parameters.

\begin{tabular}{lc}
\hline \hline Foil shape & NACA0012 \\
\hline Chord, $c$ & $0.2 \mathrm{~m}$ \\
Span (approximately) & $0.4 \mathrm{~m}$ \\
Angle & $-15^{\circ}$ to $+15^{\circ}$ \\
Gap, $\tau$ & $0-50 \mathrm{~mm}$ \\
\hline \hline
\end{tabular}




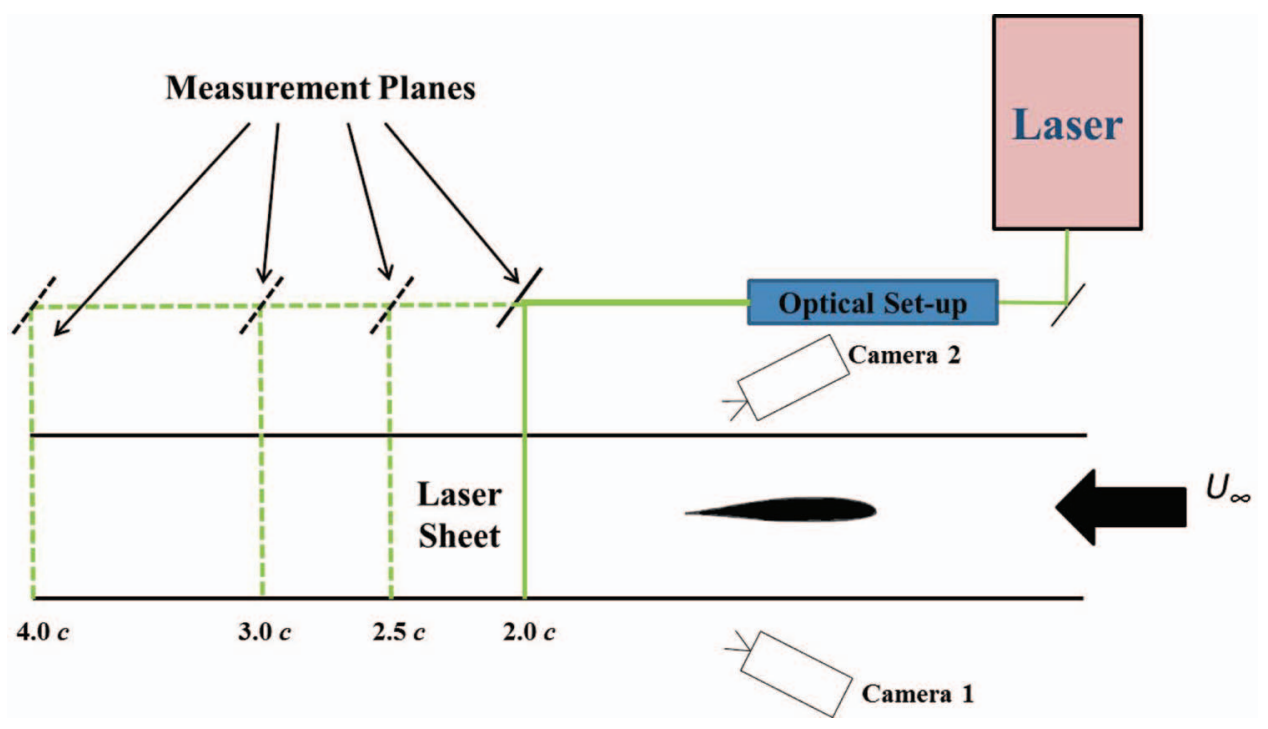

FIG. 4. Schematic of test section for PIV as seen from above.

trailing edge of the airfoils when at $0^{\circ}$ angle of attack. A range of gap sizes and angles of attack were considered. The gap size, $\tau$, ranged from $18 \mathrm{~mm}$ to $50 \mathrm{~mm}$, and the angle of attack ranged from $4^{\circ}$ to $12^{\circ}$. In the interest of brevity, all of the data presented in Sec. III correspond to a constant gap size of $30 \mathrm{~mm}$, and an angle of attack of $8^{\circ}$. 3C PIV results were obtained for all trials, but only in-plane velocity measurements are shown since the streamwise component of velocity does not aid in detecting the Crow instability.

\section{B. Vortex center detection methods}

Due to the relatively low frequency of the PIV system used $(4 \mathrm{~Hz})$ and the high expected frequency of the Crow instability $(150 \mathrm{~Hz})$, the most accurate way to detect the presence of the instability is to use the center locations of the instantaneous snapshots taken. A $45^{\circ}$ oscillation of the vortex centers is expected if the instability is present. ${ }^{4}$ In a previous study performed using the same experimental apparatus and a high frequency time resolved PIV system, the displacement of the vortex centers was found to correlate very well with the presence of the Crow instability. ${ }^{20}$ Furthermore, the vortex oscillation angles seen in that experiment are very similar to the angles observed in the present study. Such analysis, therefore, does not require time-resolved PIV, but does require a reliable method to detect the vortex center from an instantaneous PIV vector field, with limited signal-to-noise ratio.

Many different potential center detection algorithms were investigated in order to determine the most appropriate method for this study. A robust, efficient, and easily automated algorithm was desired as this method would be applied to large numbers of instantaneous PIV snapshots (1000 or 2000 images at a time). In an effort to reduce the influence of erroneous vectors, methods requiring no interpolation or calculations of derivatives were preferred. Both the $\Gamma_{1}$ and $\Gamma_{2}$ methods ${ }^{21}$ seemed to be the most promising of pre-existing methods, especially when compared to the $\mathrm{Q}$ criterion, ${ }^{22}$ and maximum vorticity methods. ${ }^{22}$ In the end, an in-house method for center detection was developed which was heavily based on the $\Gamma_{1} / \Gamma_{2}$ methods.

For the maximum vorticity method, the vorticity field was calculated for every snapshot, and the center was taken to be the point with the largest magnitude of vorticity. With this method, one erroneous vector could create a large spike in vorticity, which would cause an incorrect center to be detected. The Q method involves many gradients to be calculated, and due to noise in the PIV velocity fields, the results were not found to be reliable for this study. 


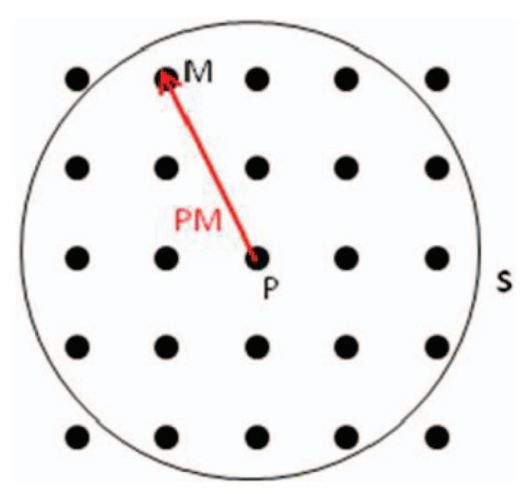

FIG. 5. Representation of the different vectors needed for the $\Gamma_{1}$ and $\Gamma_{2}$ methods.

Graftineaux defined two additional quantities that can be used to calculate vortex centers and to identify points inside of a vortex, known as $\Gamma_{1}$ (Eq. (2)) and $\Gamma_{2}$ (Eq. (3)),

$$
\Gamma_{1}(P)=\frac{1}{N} \sum_{S} \frac{\left(P M \wedge U_{M}\right) \cdot z}{\|P M\| \cdot\left\|U_{m}\right\|}
$$

where $P$ is a fixed point in the measurement domain. $S$ is a two-dimensional area surrounding $P$, referred to as the window, whose size must be defined by the user. $N$ is the number of data points $(M)$ that are located within $S$, and $U_{M}$ is the velocity vector at point $M$. Finally, $z$ is a unit vector normal to the plane of measurement. $\Gamma_{1}$ is calculated for all points in the measurement domain and then used to determine the center of the vortex. For an ideal axisymmetric vortex, the maximum value of $\left|\Gamma_{1}\right|$ is 1 . For each possible center position, the $\Gamma_{1}$ criterion calculates the degree to which the flow rotates around this point. In this work, near the vortex core, $\left|\Gamma_{1}\right|$ was found to reach values between 0.9 and 1. A diagram for this method is shown in Fig. 5.

The $\Gamma_{2}$ method is a variant of the $\Gamma_{1}$ method and the criterion for $\Gamma_{2}$ is given by Eq. (3),

$$
\Gamma_{2}(P)=\frac{1}{N} \sum_{S} \frac{\left(P M \wedge\left(U_{M}-\overline{U_{p}}\right)\right) \cdot z}{\|P M\| \cdot\left\|U_{m}-\overline{U_{p}}\right\|} .
$$

The only difference between the $\Gamma_{1}$ and $\Gamma_{2}$ criteria is that the average velocity over the window, $\bar{U}_{p}$, is subtracted away. This takes into account any uniform flow within the plane of rotation. With this method, when $\left|\Gamma_{2}\right|$ is greater than approximately $2 / \pi, P$ is assumed to represent a point in the vortex. This value for $\Gamma_{2}$ is approximately where the flow switches from being locally dominated by strain, to being locally dominated by rotation. ${ }^{21}$

In this experiment, the $\Gamma_{2}$ method was used initially instead of the $\Gamma_{1}$ method, and the center of the vortex was identified to be the point where $\left|\Gamma_{2}\right|$ was the largest. As long as the window size chosen was on the order of the calculated vortex radius, or smaller, the window size did not play a large role in determining the vortex center. This method also has the advantage that $\Gamma_{2}$ will change signs for different signs of vorticity, and no gradients need to be calculated.

Based on the $\Gamma_{2}$ method, a new center detection algorithm was developed. For each snapshot taken, the circulation was calculated at all points in the domain by using a square integration window around each point. The center was taken to be the point in the domain which had the largest circulation. A square integration window was used to avoid additional interpolations when calculating the circulation, $\Gamma=\oint u \cdot d l$. The integration must be done over a closed contour, and $u$ represents the velocity vector at any point in the domain. Circulation was calculated using a line integral instead of an area integral with vorticity in order to avoid calculating derivatives of the velocity field. Interestingly, the integration was found to smooth out some of the inherent noise in the velocity field. This method gave extremely similar results to the $\Gamma$ methods, but was computationally cheaper to perform and had a clear physical meaning.

In order to test this method, a velocity field corresponding to an ideal Lamb-Oseen vortex was generated for the same mesh spacing used in the experiment. A Lamb-Oseen vortex is an ideal 
axysymmetric vortex with a velocity profile given by Eq. (4),

$$
u_{\theta}(r)=\frac{\Gamma}{2 \pi r}\left(1-e^{-r^{2} / a^{2}}\right),
$$

where $u_{\theta}$ is the azimuthal velocity component, $\Gamma$ is the circulation of the vortex, $r$ is the distance from a point in the domain to the vortex center, and $a$ is the vortex radius. White Gaussian noise was added to this ideal profile to mimic experimental results, and the above described circulation center detection method was applied. Average values for circulation and radius were used to simulate the experimental conditions as best as possible. It was found that when the average magnitude of the white noise was $10 \%$ of the maximum vortex velocity, the average error in the center detection in $\mathrm{x}$ and y was around 0.1 grid points.

\section{EXPERIMENTAL RESULTS}

In this section the experimental results are presented. Vortex meandering, sample velocity fields, and center distributions are presented for the two configurations.

\section{A. Vortex meandering}

In order to accurately describe the physics of a system when averaging instantaneous PIV snapshots, the phenomenon of vortex meandering must be taken into account. Vortex meandering refers to the seemingly random and slow oscillation of a vortex center for a vortex that has been produced in a wind tunnel. There is neither preferred direction of oscillation nor characteristic frequency associated with this phenomenon. For any time averaged quantities calculated from PIV images, vortex meandering will cause the results to inaccurately describe the characteristics of the true vortex being studied. For example, the movement of the vortex centers will cause the vortex radius calculated from an averaged image to be larger than it should be, as the meandering effect effectively obscures the true nature of the vortex. Many attempts have been made to quantify the effect of this meandering on the vortex. After careful modeling, Devenport et al. have been able to quantify the effects of vortex meandering, showing that the vortex core radius is over predicted, the tangential velocity is under predicted, and the velocity deficit in the axial direction is also under predicted if this effect is not taken into account. ${ }^{23}$ Although the Crow instability has a preferred direction of oscillation for the vortex centers, the overall effect of this instability on calculated averaged PIV quantities will be similar to the effect of vortex meandering.

Figure 6 shows two sample averaged in-plane vector fields measured experimentally. The right image shows two counter-rotating vortices, while the left image shows one vortex close to the splitter
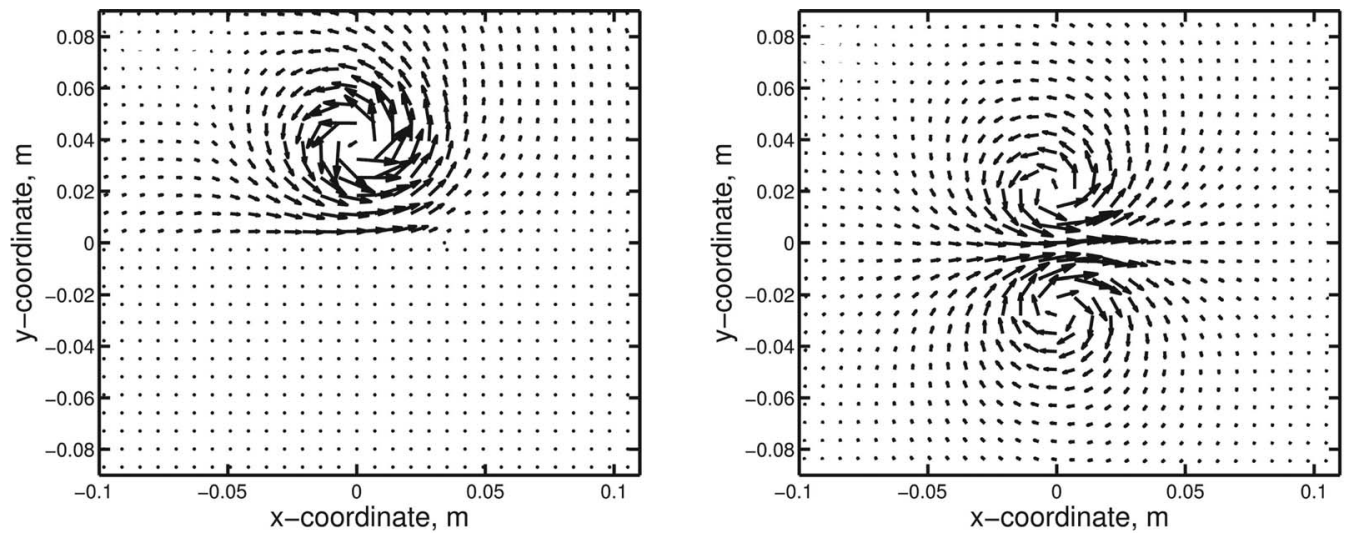

FIG. 6. Sample averaged in-plane velocity vectors taken at $2.5 c$ downstream, $\tau=30 \mathrm{~mm}$, and a free stream velocity of 39.5 m/s. Left: C1 (1000 images averaged). Right: C2 (2000 images averaged). Some vectors have been removed for clarity. 


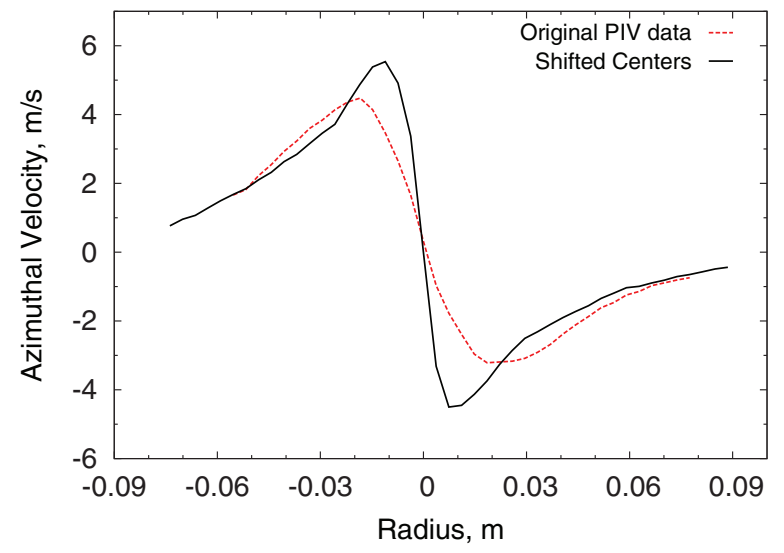

FIG. 7. Results comparing shifted and non-shifted centers for $2.5 c$ downstream, $\tau=30 \mathrm{~mm}$.

plate. Neither the effect of vortex meandering nor the Crow instability has been taken into account with these images.

In order to quantify these two effects, all of the instantaneous snapshots were shifted so that each image was centered on the same grid point. Next, the velocity field was averaged in an attempt to analyze the true characteristics of the vortex. Figure 7 shows how the oscillating center of the vortex affects the values calculated from an averaged velocity field. Here, a horizontal cut is taken along the centerline of the vortex from the averaged PIV field. The y-coordinate is held constant in the plane and taken at the center of the vortex, and the vertical component of velocity is plotted. For the data represented by the dotted line, no corrections were made to the averaged velocity field calculated. For the solid line data, each individual snapshot was shifted so that all calculated centers lined up with each other before the average velocity field was calculated. The effect of both the Crow instability (which was present in this trial) and vortex meandering can clearly be seen. By shifting all vortex centers, the vortex radius has been decreased, and the maximum azimuthal velocity has been increased, consistent with predictions from Devenport et al. ${ }^{23}$ Farther, downstream re-centering all of the images has a larger effect, as the oscillations from both vortex meandering and the Crow instability increase in magnitude. When this algorithm was applied to circulation calculations, the circulation of a vortex was observed not to change if the circulation was calculated far enough away from the vortex center.

\section{B. Vortex dynamics}

The presence of the Crow instability may be detected with two approaches: first, by studying the preferred direction of oscillation; second, by evaluating the magnitude of the oscillations. Figure 8 shows a contour plot representing the frequency at which the center of the vortex falls on a specific grid point for one data series. Once again, the presence of the Crow instability is determined by a preferred angle of oscillation of the vortex centers, in this case a $45^{\circ}$ angle. ${ }^{4}$ In the left figure, the two-vortex configuration is shown where the Crow instability is clearly present; the vortex centers oscillate along a preferred direction and with an angle close to $45^{\circ}$. In the right figure, the one-vortex configuration is shown where the Crow instability is not present; the vortex center oscillates with no preferred direction.

While it is possible to identify visually whether or not the Crow instability is present, a more quantitative analysis was used to estimate the preferred angle of oscillation. More precisely, a principal component analysis (PCA) was used in order to determine the primary direction of oscillation of the vortex centers. ${ }^{24}$ These results are summarized in Table II.

The first quantity of interest is the angle corresponding to the most probable direction of oscillation, as defined in Fig. 8 (left). Although the predicted theoretical value of $45^{\circ}$ is not observed, the two angles do remain fairly constant, at approximately $70^{\circ}$ and $60^{\circ}$. Similar angles were observed 

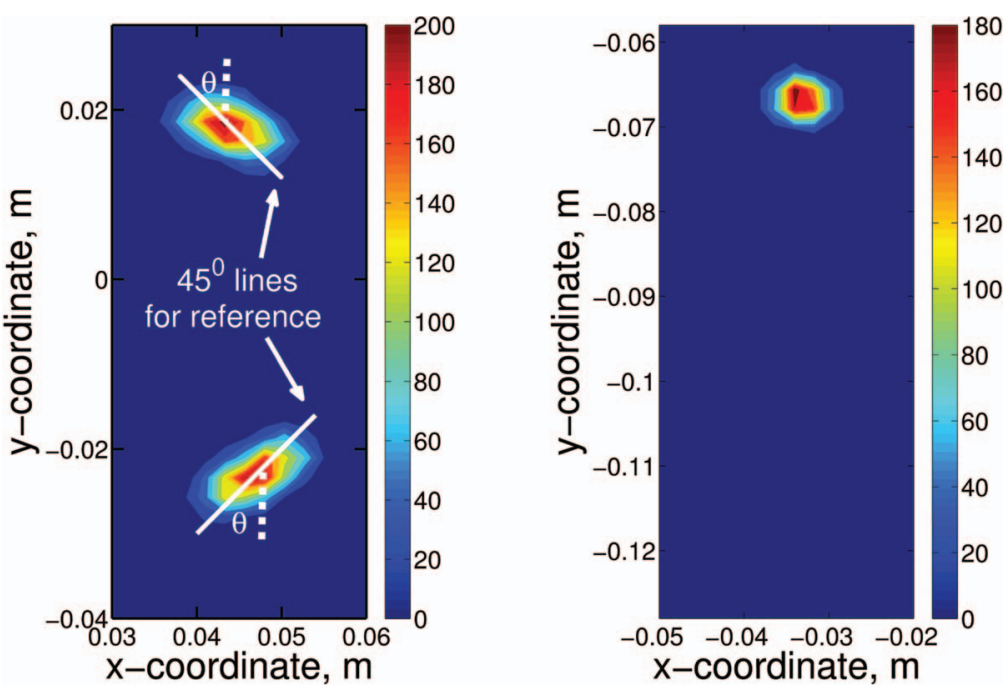

FIG. 8. Contour plots showing the distribution of centers for $\tau=30 \mathrm{~mm}, 2.5 \mathrm{c}$ downstream and a free stream velocity of $39.5 \mathrm{~m} / \mathrm{s}$. Left: C2 (2000 images used). Right: C1 (1000 images used). White lines have been drawn at $45^{\circ}$ manually showing the predicted preferred direction of the center oscillation. The colors refer to counts of how many times the same center positions were found during one experiment.

in a previous study with a high frequency time resolved PIV system, when the Crow instability was observed. ${ }^{20}$ The deviation from the theoretical value may be attributed to the presence of oscillations other than the most unstable one. The second quantity of interest is the normalized PCA eigenvalue and is a measure of how much (percentage) of the center oscillations are in this primary direction. The relatively high eigenvalues indicate that the majority of movement of the vortex centers occurs along this direction, which is attributed to the Crow instability. Movement in other directions can be attributed to vortex meandering, turbulence, etc.

The same analysis was performed for the $\mathrm{C} 1$ configuration and the results showed no preferred direction of oscillation (first and second eigenvalues were approximately equal).

If the Crow instability is not present, the center oscillations can be attributed to vortex meandering effects. ${ }^{23}$ When the Crow instability is present, the centers are expected to oscillate much more than when the Crow instability is not present. One way to estimate this is to calculate an average distance from the mean center position, using

$$
d=\frac{1}{N} \sum_{i=1}^{N} \sqrt{\left(x_{i}-x_{\text {mean }}\right)^{2}+\left(y_{i}-y_{\text {mean }}\right)^{2}} .
$$

Here, $x_{i}$ and $y_{i}$ refer to the coordinates of the centers found from the circulation method for each snapshot, and $N$ images have been taken at a particular downstream location. The evolution of this

TABLE II. Results from principal component analysis.

\begin{tabular}{lccc}
\hline \hline Downstream location & Vortex location & Angle (deg) & Normalized first eigenvalue \\
\hline $2.0 c$ & Top & 70 & 0.75 \\
$2.0 c$ & Bottom & 60 & 0.78 \\
$2.5 c$ & Top & 70 & 0.77 \\
$2.5 c$ & Bottom & 62 & 0.80 \\
$3.0 c$ & Top & 70 & 0.75 \\
$3.0 c$ & Bottom & 60 & 0.77 \\
$4.0 c$ & Top & 76 & 0.66 \\
$4.0 c$ & Bottom & 66 & 0.69 \\
\hline \hline
\end{tabular}




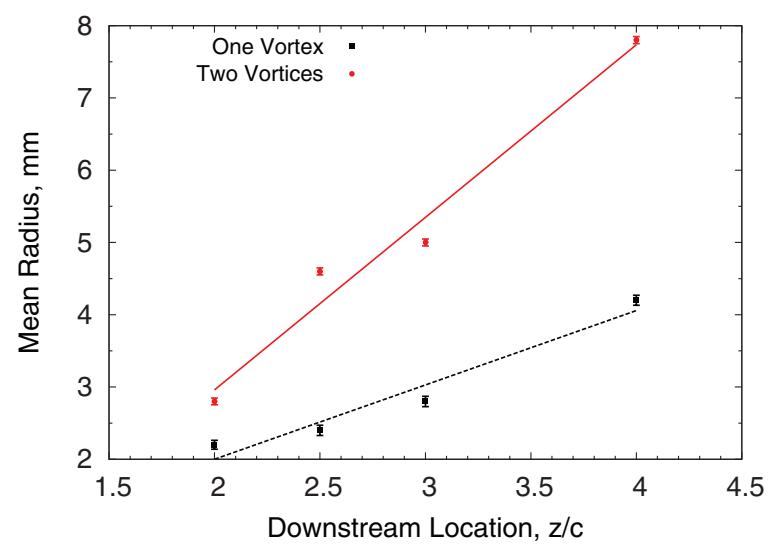

FIG. 9. Average distance of the instantaneous vortex centers to the mean center $(\tau=30 \mathrm{~mm})$. When two vortices are present, the radius for the top and bottom vortices are averaged.

mean distance as a function of downstream location is shown in Fig. 9 for both configurations. As expected, this mean distance increases with downstream distance. The overall growth observed is much larger for the $\mathrm{C} 2$ configuration than for the $\mathrm{C} 1$ configuration, further confirming that the Crow instability is not present in the $\mathrm{C} 1$ configuration.

\section{NUMERICAL SIMULATIONS}

In this section, the numerical framework and the simulation results are presented both for the one-vortex and the two-vortex configurations. The objective is not to reproduce the experimental results, but to provide an independent, yet complimentary, investigation of the effect of the splitter plate on the dynamics of the vortex.

\section{A. Numerical framework}

Remaining true to Crow's original work, ${ }^{4}$ the numerical simulations performed are temporal in nature with periodic boundary conditions in the axial direction (along the vortex axis). This means that the physics associated with the initial vortex roll-up and the axial boundary layer are not included in the numerical simulations. It is not necessary to reproduce these two experimental phenomena in order to study the formation of the Crow instability. Furthermore, the circulation of each vortex was observed to decrease at all measurement locations (Fig. 10). In other words, the roll-up process is already completed at $2.0 c$ downstream of the trailing edge of the foils. Finally, the circulation

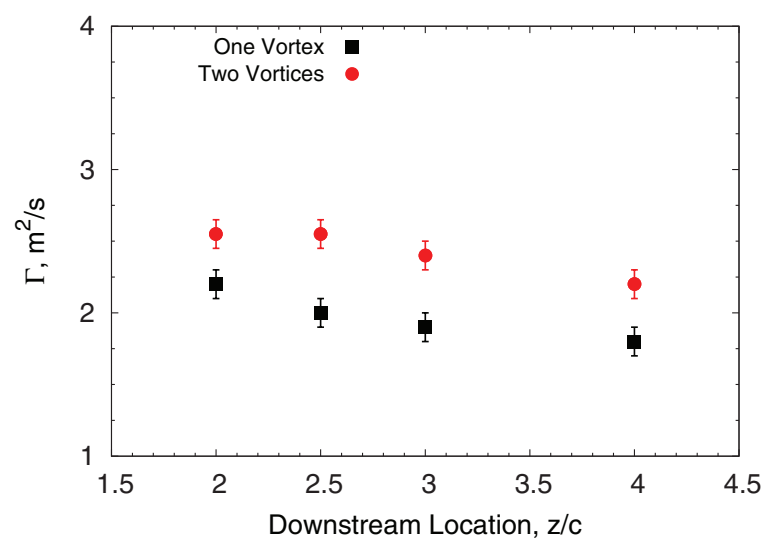

FIG. 10. Downstream evolution of circulation for both the $\mathrm{C} 1$ and $\mathrm{C} 2$ configurations. For the $\mathrm{C} 2$ configuration, an average circulation is calculated from the two vortices. 
contained in the axial boundary layer remains very small (estimated at $0.02 \%$ of that of the primary vortex), and therefore can be neglected without affecting the development of the Crow instability.

In all of the numerical simulations, a high-order, fully conservative, finite difference scheme for the Navier-Stokes equations was used, called NGA. ${ }^{25}$ From previous studies investigating the interactions of a vortex and a wall, it had been determined that using a 4th order accurate scheme for both the convective and viscous terms was sufficient to accurately capture the full physics of the flow.

\section{B. Two vortex simulations}

To study the interactions of two counter-rotating vortices, the numerical simulations are initialized with two counter-rotating Lamb-Oseen vortices. In addition, a velocity equal and opposite to the speed of the two-vortex system is superimposed to ensure that the vortices remain stationary during the simulations. Figure 11 shows a comparison between an experimentally measured velocity field and the initial velocity field used in the numerical simulations. The Lamb-Oseen vortex shape was found to reproduce the experimental vortices well.

The first 3D simulation performed, consisted of two straight counter-rotating Lamb-Oseen vortices in a periodic domain, with $L_{x}=L_{y} \sim 20 b . L_{x}$ and $L_{y}$ refer to the length of the numerical domain in both the $x$ and $y$ directions, respectively. In the wind tunnel, the time taken by the mean flow to travel from the trailing edge of the airfoils to the final plane of measurement is approximately $0.02 \mathrm{~s}$. The vortices were found to remain stationary and unperturbed for the entire numerical run time of $0.1 \mathrm{~s}$, which was much larger than the expected time needed for the Crow instability to develop. This proved that the numerical approach did not introduce any unphysical oscillations, and therefore was well suited for the present investigation.

In order to confirm that the Crow instability could be observed numerically, a sinusoidal perturbation corresponding to the theoretical most amplified wavelength was added to the initial conditions ( $k b \sim 1$, where $k$ is the axial wave number, and $b$ is the vortex separation distance). The center of the vortex was varied in the y direction (perpendicular to the vortex), with a maximum disturbance of $10 \%$ of the vortex radius. Figure 12 shows the evolution of the system in time. The initial perturbations are continuously amplified until the vortices eventually connect and breakup. It is possible to see a preferred angle of oscillation, and there is a clear plane of symmetry between the vortices.

In order to quantify the evolution of the Crow instability, we performed a similar analysis to that which was performed earlier with the experimental data. The same center-detection method that was used to analyze the PIV data is applied to each z-plane cut for a numerical simulation. Figure 13 shows the temporal evolution of the principal angle calculated. The PCA is very sensitive to small perturbations of the vortex and can calculate the correct angle of oscillation even at very

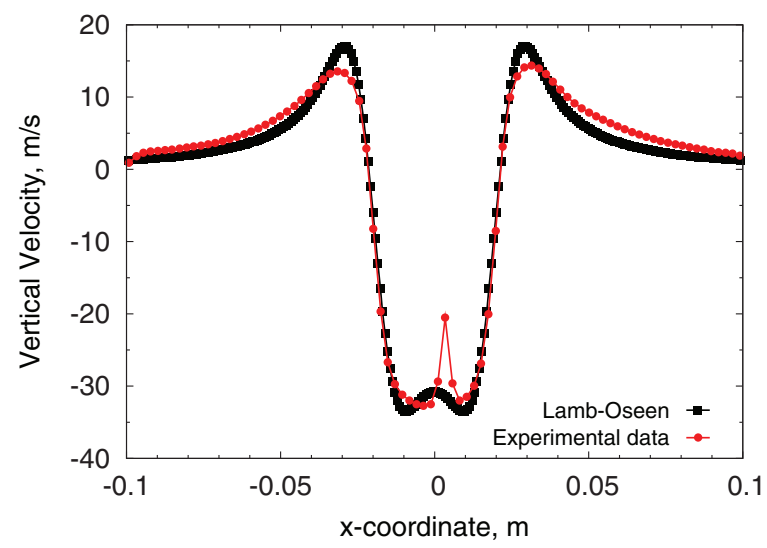

FIG. 11. Comparison between an ideal Lamb-Oseen velocity field and the experimental data. 

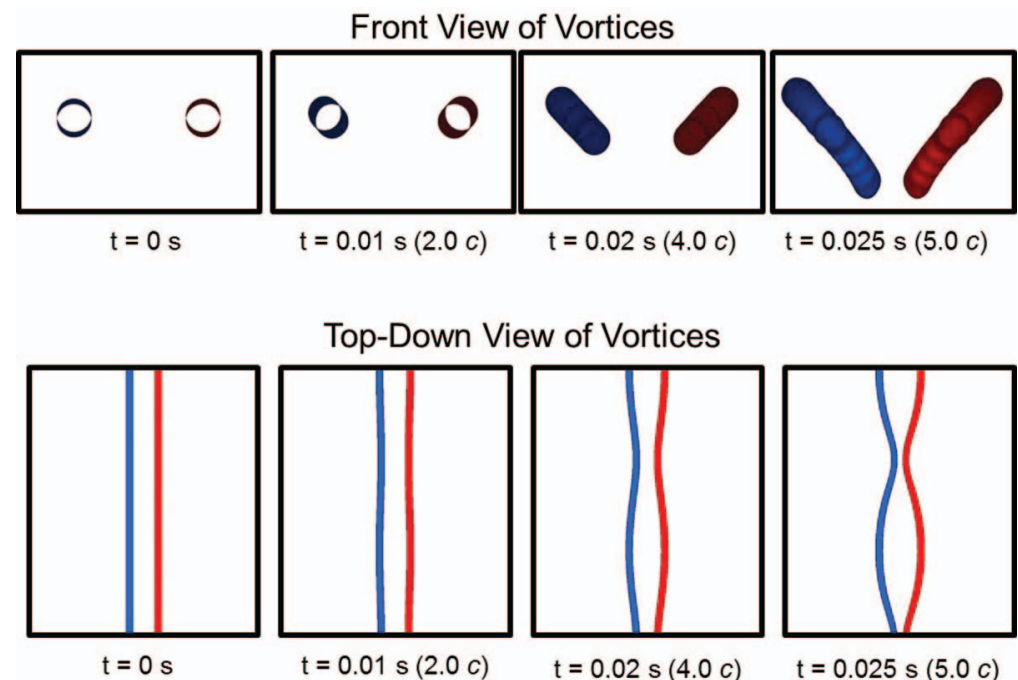

\section{Top-Down View of Vortices}
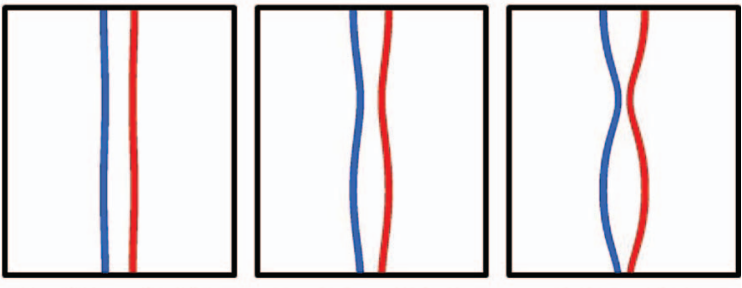

$\mathrm{t}=0.01 \mathrm{~s}(2.0 \mathrm{c})$

$\mathrm{t}=0.02 \mathrm{~s}(4.0 \mathrm{c})$

$t=0.025 \mathrm{~s}(5.0 \mathrm{c})$

FIG. 12. Temporal evolution of the two-vortex system under an ideal sinusoidal initial perturbation.

small times. At the beginning of the simulation, the $0^{\circ}$ angle is consistent with the direction of the initial perturbation imposed. After an initial transient period, the average angle becomes $44^{\circ} \pm 5^{\circ}$, as expected for the most unstable wavelength $\left(48^{\circ}\right)$.

\section{One vortex and a wall configuration}

The second set of simulations was performed with a Lamb-Oseen vortex interacting with a wall. The initial velocity profile used for these simulations was based on a Lamb-Oseen vortex and an image Lamb-Oseen vortex located beneath the wall. Once again, the numerical approach was decomposed into two steps.

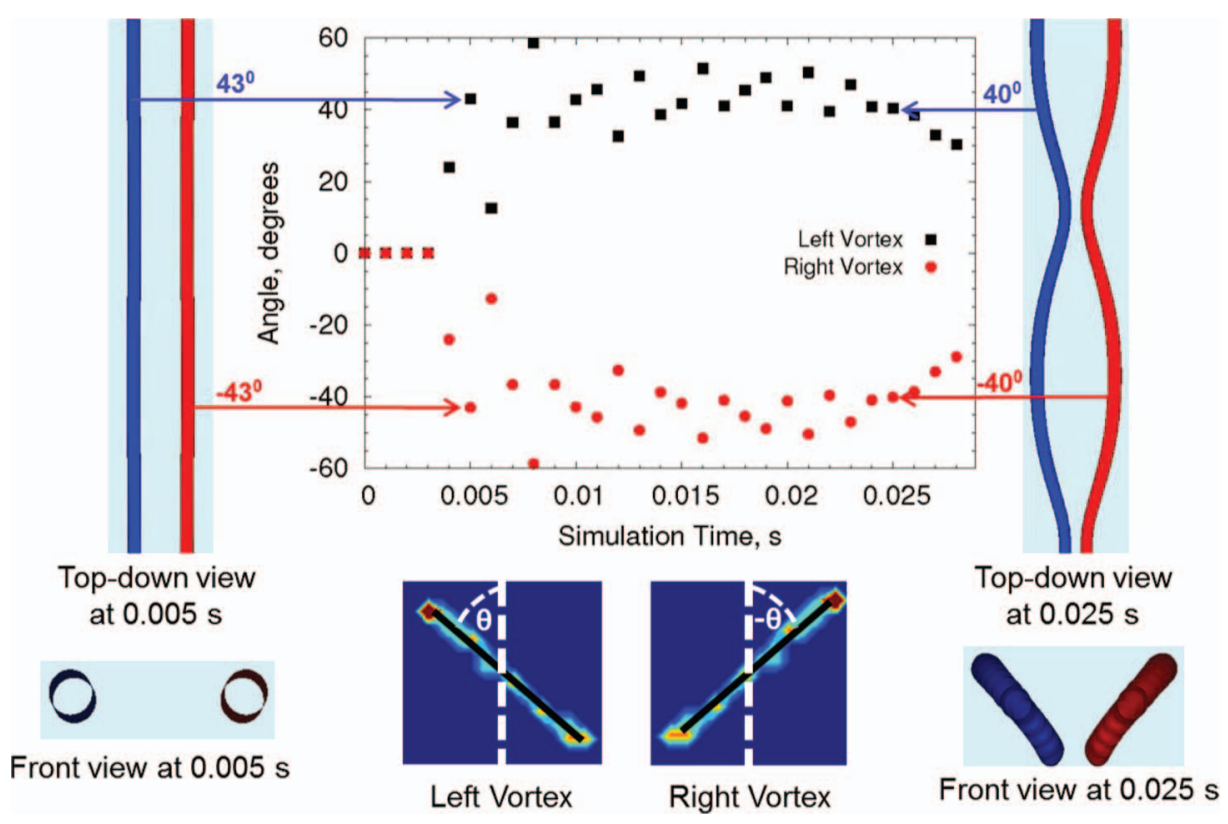

FIG. 13. Temporal evolution of the principal angle for the two vortices. Even when the perturbations of the vortices are small $(t=0.005 \mathrm{~s})$, the PCA analysis is still able to pick up an angle close to $45^{\circ}$. 
Evolution of Vortex and a Wall System

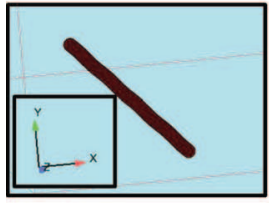

$t=0 s$

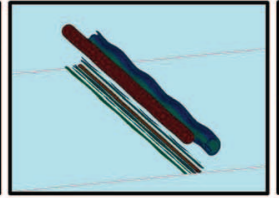

$t=0.01 s(2.0 c)$

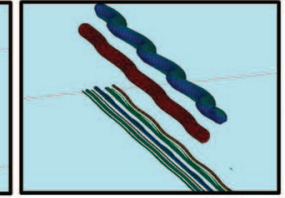

$t=0.02 s(4.0 c)$

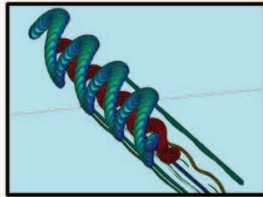

$t=0.03 s(6.0 c)$

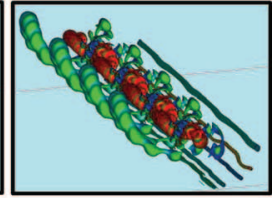

$t=0.04 s(6.0 c)$

FIG. 14. Temporal evolution of the one-vortex system under an ideal sinusoidal initial perturbation.

First, a slip condition in $x$ and a no penetration condition in $y$ was used along the wall at the bottom of the domain to mimic an inviscid wall and to see if the Crow instability would develop as it previously did with the two vortices. We observed that the Crow instability did in fact develop in the same manner that it did before. Once again, the domain was chosen to be large enough in comparison to the size of the vortex ( $L_{x} \sim 75 a, L_{y} \sim 65 a$, where $a$ is the Lamb-Oseen vortex radius), and large in comparison to the separation distance between the vortex and the wall, which was $\sim 4 a$, to ensure that the boundary conditions had no effect on the simulation. The results confirmed that, in the inviscid limit, the $\mathrm{C} 1$ and $\mathrm{C} 2$ configurations are equivalent.

When a no-slip condition was added to the wall in the $x$ direction, the simulations became very sensitive to the initial velocity profile used next to the wall at the bottom of the numerical domain. When no attempt is made to account for a boundary layer in the initialization process, unphysical oscillations are generated next to the wall due to the sharp discontinuity in velocity. These oscillations are then convected away from the wall by the rotation of the vortex. After many different attempts, it was found that a "linear" velocity profile with a boundary layer height of $18 \%$ of the spacing between the vortex center and the wall reproduced the experimental results most closely.

The evolution of the one-vortex system is fundamentally different than the evolution of the two-vortex system. Figure 14 shows how the simulation evolves in time. Secondary vortices of opposite signed vorticity are rolled-up away from the wall $(t=0.01 \mathrm{~s})$, and begin to rotate around the primary vortex $(t=0.02 \mathrm{~s}$ and $t=0.03 \mathrm{~s})$. The initial perturbations are amplified and a highly 3D turbulent flow field is produced $(t=0.04 \mathrm{~s})$. Due to resolution limits, a Lagrangian dynamic subgrid scale model is used to represent the effects of the unresolved small-scale fluctuations on the dynamics of the large-scale structures. ${ }^{26}$ As time progresses, a complex interaction is seen between the primary vortex and the secondary vortex rolled up from the wall.

In summary, in the presence of a wall, the primary vortex is not observed to break down due to its mirror image on the other side of the wall, but due to the roll-up of a secondary vortex. In other words, the Crow instability is not observed for a viscous wall.

\section{DISCUSSIONS}

In this section, the results from the experimental and numerical studies are summarized and an explanation is provided for the different behaviors observed in the two configurations.

\section{A. Presence of the Crow instability}

Many differences were observed between the single-vortex $(\mathrm{C} 1)$ and the two-vortex $(\mathrm{C} 2)$ configurations, and these differences contributed to detecting the Crow instability only for the $\mathrm{C} 2$ configuration and not for the $\mathrm{C} 1$ configuration. Whether or not the Crow instability is present in a flow follows a trend based on viscosity.

- With two vortices present, the Crow instability is present (experiments and simulations). 

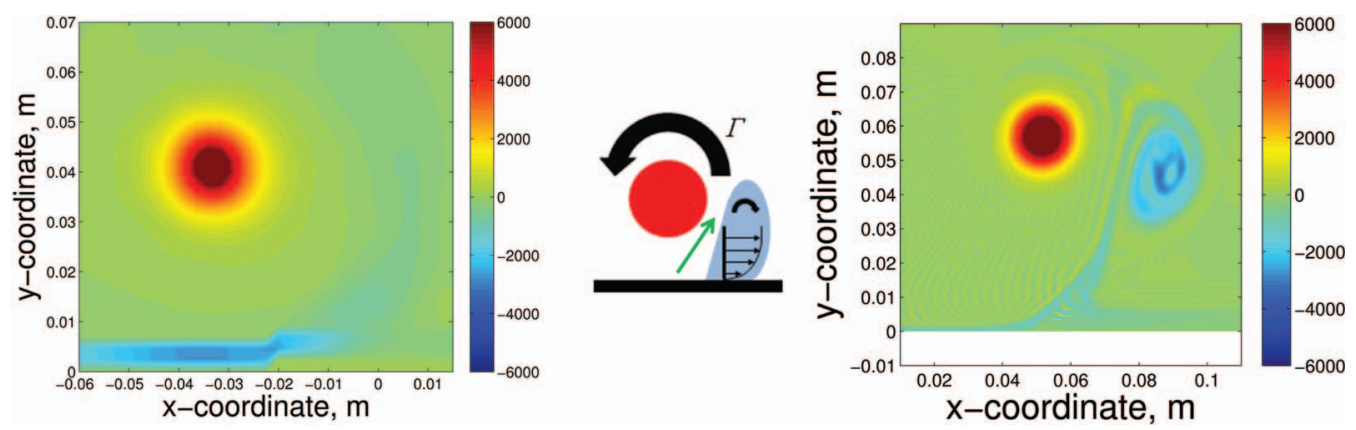

FIG. 15. Contour plot of vorticity $\left(\mathrm{s}^{-1}\right)$ for a gap size of $30 \mathrm{~mm}$ and at a location $2.5 c$ downstream (Left: experiment, Right: simulation). The opposite signed vorticity from the boundary layer has been convected away from the wall by the vortex. Center: schematic diagram explaining the origins of the rebound effect and the streamwise boundary layer. The whole system is convected in the direction of the straight arrow.

- With one vortex in the vicinity of a slip wall (inviscid boundary condition-simulation), the Crow instability is also present.

- With one vortex in the vicinity of a no slip wall (viscous boundary condition-simulation and experiment), the Crow instability is not observed.

These observations are supported by both experimental work and numerical simulations.

It may be argued that the computations never included the axial boundary layer, and hence do not compare to the experiments. However, in light of the current numerical results, it is doubtful that adding an additional (axial) boundary will promote the growth of the Crow instability, when the cross-stream boundary layer suppresses the instability.

\section{B. Effects of viscosity}

The effect of viscosity along the splitter plate causes the single-vortex configuration to evolve very differently from the two-vortex configuration.

Due to the no-slip condition, a boundary layer along the splitter plate in the cross-stream direction is created as well as a boundary layer in the streamwise direction. This in turn creates a region of opposite signed vorticity near the wall, which is convected away from the wall by the vortex. These two regions of opposite signed vorticity cause a rebound effect, increasing the distance between the vortex and the wall (increase in $b$ ), as the vortex travels downstream. These effects are shown in Fig. 15. While the experimental measurements and numerical results differ slightly on the magnitude of these phenomena, both of them predict consistently the formation of a secondary vortex and a rebound of the primary vortex. The numerical results and experimental data are used for qualitative comparisons only, as the complex roll-up process that generates the vortex in the experiment is not incorporated into the numerical simulations. However, none of these two phenomena were observed in the single-vortex configuration.

\section{Characteristic time}

A fundamental difference between the two configurations is the discrepancy in characteristic times for the Crow instability to develop $\left(t_{b}^{*}\right)$. The time scale associated with the Crow instability is given by

$$
t_{b}=\frac{2 \pi b^{2}}{\Gamma} .
$$

Here, $b$ is the distance between the vortex centers. This time is generally used only as an estimate for the time required for the Crow instability to develop. In practice, several times $t_{b}$ are necessary before the instability may be detected. ${ }^{1}$ This parameter is often taken to be the most important 




FIG. 16. Evolution of the characteristic time vs downstream location $(\tau=30 \mathrm{~mm})$.

factor to compare different configurations and analyze the dynamics of different vortex systems. A non-dimensional version of Eq. (6) is given by

$$
t_{b}^{*}=t_{b} \frac{U_{\infty}}{c} .
$$

When this quantity is smaller than unity $\left(t_{b}^{*}<1\right)$, the Crow instability has time to form. When the quantity is much greater than unity, the Crow instability is unlikely to form. Figure 16 provides a comparison of the non-dimensional times for the $\mathrm{C} 1$ and $\mathrm{C} 2$ configurations. The large difference in $t_{b}^{*}$ between the two configurations explains why the dynamics of the two systems are so different. The two-vortex configuration has time to develop the Crow instability; whereas the single vortex does not.

In an attempt to promote the Crow instability in the $\mathrm{C} 1$ configuration, the experimental parameters were varied in an attempt to reduce $t_{b}^{*}$ and match the $t_{b}^{*}$ for the two vortex case shown in Fig. 8. Unfortunately, matching the characteristic times between the two configurations proved to be much more challenging than expected. With the experimental apparatus, it was possible to change both the angle of attack as well as the gap size. However, increasing the gap size increased $b$ but also increased $\Gamma$. The opposite was seen if the gap size was decreased. The angle of attack did not have a large effect on the characteristic time. This led to a characteristic time that was fairly constant for the $\mathrm{C} 1$ configuration, and not low enough for the Crow instability to have time to develop.

\section{Boundary layer}

Another factor which contributes to the increase in $b$ in the $\mathrm{C} 1$ configuration downstream is simply the increase in size of the streamwise boundary layer. A turbulent boundary layer was initiated at the leading edge of the splitter plate. Hence, the following correlation may be used to model the growth of the turbulent boundary layer:

$$
\delta=\frac{0.37 z}{\left(\frac{U_{\infty} z}{v}\right)^{\frac{1}{5}}} .
$$

In order to analytically relate the change in $t_{b}^{*}$ due to the distance travelled downstream, it is assumed that the change in characteristic time is solely caused by a change in $b$, and this change in $b$ is caused by the boundary layer growth in the streamwise direction. Combining Eqs. (6)-(8), it can be shown that $\Delta t_{b}^{*} \sim \Delta z^{*}$, where $z^{*}=z / c$. Figure 16 shows that the growth of the characteristic time seems to be linear with downstream distance. This trend is the same as the growth predicted by the simplified boundary layer model. Most likely, the increase in characteristic time is a combination of the boundary layer effect, the rebound effect, and the decrease in circulation as the vortex travels downstream. 
This implies that if the Crow instability is not present immediately in the flow, it may not develop at any downstream locations, due to the continual increase in the boundary layer thickness and hence the characteristic time.

\section{CONCLUSIONS}

A counter-rotating vortex pair (C2) and a vortex interacting with a wall (C1) have been analyzed. Experimental measurements and numerical simulations were performed to determine if the Crow instability was present in these configurations. With the $\mathrm{C} 2$ configuration, it was possible to observe the Crow instability, while the Crow instability was not observed with the C1 configuration. The increase in characteristic time for the Crow instability as one travels downstream in the $\mathrm{C} 1$ configuration implies that if the Crow instability is not present initially, it may never develop downstream. The movement of the vortex away from the wall is mainly due to the growth of the streamwise boundary layer. The way in which a single vortex in the vicinity of a wall breaks up is fundamentally different than the breakup of two counter-rotating vortices. Numerical simulations have re-enforced the experimental results, illustrating the fundamental differences in the two systems and that these differences originate from the viscous forces acting at the wall. In summary, while a single vortex and a wall configuration is equivalent to a two vortex setup from an inviscid point of view, the viscous forces at the wall render these two setups fundamentally different.

\section{ACKNOWLEDGMENTS}

J.R. would like to thank the Dual Masters Program between the California Institute of Technology and Ecole Polytechnique, and the Partner University Fund for funding him while performing the experimental component of this research. The authors would like to thank members of ONERA DAFE, Meudon, for their help with the experiments and the post-treatment of the experimental data.

\section{APPENDIX: PIV CHARACTERISTICS}

Table III gives details about the PIV system used.

TABLE III. PIV characteristics.

\begin{tabular}{lc}
\hline \hline Type of PIV & Stereo(3C) \\
\hline Laser & Nd:YAG Thales PVL 400/TS \\
Cameras & LA vision imager intense \\
Frequency & $4 \mathrm{~Hz}$ \\
Image size (pixels) & $1376 \times 1040$ \\
Camera focal length & $50 \mathrm{~mm}$ \\
Conversion & 5.0 pixels/mm \\
Time between images & $30 \mu \mathrm{s}$ \\
Laser sheet thickness (usable) & $2 \mathrm{~mm}$ \\
Pixel shift in free stream & 4.5 \\
Vector spacing & $2.4 \mathrm{~mm}$ \\
Vortex diameter/spacing & $\sim 10$ \\
Images per trial & 1000 or 2000 \\
Seeding particles & Magnum smoke generator \\
Software used & FOLKI PIV (Ref. 27) \\
\hline \hline
\end{tabular}

${ }^{1}$ P. R. Spalart, “Airplane trailing vortices,” Annu. Rev. Fluid Mech. 30, 107 (1998).

${ }^{2}$ L. Jacquin, D. Fabre, P. Geffroy, and E. Coustols, "The properties of a transport aircraft wake in the extended near field-An experimental study,” AIAA Paper 2001-1038, 2001. 
${ }^{3}$ C.-Y. Tsai and S. Widnall, "The stability of short waves on a straight vortex filament in a weak externally imposed strain field," J. Fluid Mech. 73, 721 (1976).

${ }^{4}$ S. C. Crow, "Stability theory for a pair of trailing vortices," AIAA Paper No. 0001-1452, 1970.

5 J. D. Denton, "Loss mechanisms in turbomachines," J. Turbomach. 115, 621 (1993).

${ }^{6}$ B. Lakshminarayana and J. Horlock, "Tip-clearance flow and losses for an isolated compressor blade," Aeronautical Research Council Reports and Memoranda 3316, 1963.

${ }^{7}$ J. Storer and N. Cumpsty, "Tip leakage flow in axial compressors," J. Turbomach. 113, 252 (1991).

${ }^{8}$ G. Chen, E. Greitzer, C. Tan, and F. Marble, "Similarity analysis of compressor tip clearance flow structure," J. Turbomach. 113, 260 (1991).

${ }^{9}$ V. Brion, "Stabilité des paires de tourbillons contra-rotatifs: application au tourbillon de jeu dans les turbomachines," Ph.D. dissertation (Mécanique Département, ONERA and Ecole Polytechnique, Meudon, France, 2009).

${ }^{10} \mathrm{~J}$. Bae, "Active control of tip clearance flow in axial compressors," Ph.D. dissertation (Massachusetts Institute of Technology, 2001).

${ }^{11}$ W. J. Devenport, K. S. Wittmer, C. Muthanna, S. Bereketlab, and J. Moore, "Turbulence structure of a tip-leakage vortex wake," AIAA Paper 1997-440, 1997.

${ }^{12}$ R. Ma and W. J. Devenport, "Tip gap effects on the unsteady behavior of a tip leakage vortex," AIAA Paper No. 0001-1452, 2007.

${ }^{13}$ M. Inoue and M. Furukawa, "Physics of tip clearance flow in turbomachinery," J. Turbomach. 111, 250 (1989).

${ }^{14}$ K. Yamada, M. Furukawa, M. Innoue, and K. Funazaki, "Numerical analysis of tip leakage flow field in a transonic axial compressor rotor," International Gas Turbine Congress, Tokyo, TS-030, November 2003.

${ }^{15}$ D. You, M. Wang, P. Moin, and R. Mittal, "Effects of tip-gap size on the tip-leakage flow in a turbomachinery cascade," Phys. Fluids 18, 105102 (2006).

${ }^{16}$ O. Boulon, M. Callenaere, J. P. Franc, and J. M. Michel, "An experimental insight into the effect of confinement on tip vortex cavitation of an elliptical hydrofoil," J. Fluid Mech. 390, 1 (1999).

${ }^{17}$ W. J. Devenport, J. S. Zsoldos, and C. M. Vogel, "The structure and development of a counter-rotating wing-tip vortex pair," J. Fluid Mech. 332, 71 (1997).

${ }^{18}$ A. Heyes, R. Jones, and D. Smith, "Wandering of wing-tip vortices," in Proceedings of the 12th International Symposium on Applications of Laser Techniques to Fluid Mechanics (Lisbon, Portugal, 2004), p. 12.

${ }^{19}$ H. Schlicting, Boundary Layer Theory (McGraw-Hill, New York, 1979).

${ }^{20}$ V. Brion and L. Jacquin, "Measurements of a turbulent vortex pair using time resolved PIV," AIAA Paper 2011-3892, 2011.

${ }^{21}$ L. Graftieaux, M. Michard, and N. Grosjean, "Combining PIV, POD and vortex identification algorithms for the study of unsteady turbulent swirling flows," Meas. Sci. Technol. 12, 1422 (2001).

${ }^{22}$ J. Jeong and F. Hussain, "On the identification of a vortex," J. Fluid Mech. 285, 69 (1996).

${ }^{23}$ W. J. Devenport, M. C. Rife, S. I. Liapis, and G. J. Follin, “The structure and development of a wing-tip vortex," J. Fluid Mech. 312, 67 (1996).

${ }^{24}$ R. A. Johnson and D. W. Wichern, Applied Multivariate Statistical Analysis, 5th ed. (Prentice Hall, New Jersey, 2002), Chap. 8.

${ }^{25}$ O. Desjardins, G. Blanquart, B. Balarac, and H. Pitsch, "High order conservative finite difference for variable density low Mach number turbulent flows," J. Comput. Phys. 227, 7125 (2008).

${ }^{26}$ C. Meneveau, T. S. Lund, and W. H. Cabot, "A Lagrangian dynamic subgrid-scale model of turbulence," J. Fluid Mech. 319, 353 (1996).

${ }^{27}$ F. Champagnat, A. Plyer, G. Le Besnerais, B. Leclaire, S. Davoust, and Y. Le Sant, "Fast and accurate PIV computation using highly parallel iterative correlation maximization," Exp. Fluids 50, 1169 (2011). 